\title{
ENERGY EFFICIENT HIERARCHICAL CLUSTER-BASED ROUTING FOR WIRELESS SENSOR NETWORKS
}

\author{
Shideh Sadat Shirazi, Aboulfazl Torqi Haqiqat \\ Faculty of Computer and Information Technology Engineering, \\ Qazvin Branch, Islamic Azad University, Qazvin, Iran \\ Shirazi.shidehegmail.com \\ At_haghighat@yahoo.com
}

\begin{abstract}
In this paper we propose an energy efficient routing algorithm based on hierarchical clustering in wireless sensor networks (WSNs).This algorithm decreases the energy consumption of nodes and helps to increase the lifetime of sensor networks. To achieve this goal, this research network is divided into 4segments that lead to uniform energy consumption among sensor nodes. We also propose a multi-step clustering method to send and receive data from nodes to the base station. The simulation results show that our algorithm is better than existing algorithms in terms of saving energy, balancing energy dissipation and prolonging network lifetime.
\end{abstract}

\section{KEYWORDS}

Wireless sensor networks, energy efficient, data aggregation algorithms, clustering.

\section{INTRODUCTION}

In recent years, many ways of routing protocols based on clustering have been proposed. Clusterbased data aggregation algorithms are the most popular ones because they have the advantages of high flexibility and reliability. Recent advances in wireless communications and electronics have led to the development of WSNs, which are composed of many small-size, low-cost, low-power and multifunctional sensor nodes [1]. The process of aggregating the data from multiple nodes to eliminate redundant transmission and provide fused data to the BS - the so-called data aggregation - is considered as an effectual technique for WSNs to save energy [2]. The most popular data aggregation algorithms are cluster-based data aggregation algorithms, in which the nodes are grouped into clusters; each cluster consists of a cluster head $(\mathrm{CH})$ and some members, each of them transmitting data to its $\mathrm{CH}$. Then, each $\mathrm{CH}$ aggregates the collected data and transmits the fused data to the BS.

Natarajan Meghanathan et al. (Eds) : ACSIT, FCST, ITCA, CoNeCo - 2015

pp. 61-69, 2014. (C) CS \& IT-CSCP 2015

DOI : $10.5121 /$ csit.2015.51207 
Unbalanced energy dissipation is an inherent problem of cluster-based WSNs. Some nodes drain their energy faster than others which results in an earlier failure of network. Some researchers have studied this problem and proposed their algorithms, which have both advantages and disadvantages. Our motivation is to propose a novel solution to this problem in the cluster-based and homogeneous WSNs, in which the CHs transmit data to the BS by one-hop communication, with an objective of balancing energy consumption by an energy efficient way and, thus, prolonging network lifetime.

\section{RELATED WORK}

Some of these other algorithms which are related to our method will be discussed briefly. Most of the routing algorithms are based on a LEACH clustering algorithm; the algorithm is implemented to improve the routing. The LEACH algorithm was proposed in 2000 [3] of the random rotation techniques to select the cluster head node in the network uses. LEACH performances, in turn, are organized so that each turn consists of a setup phase and a maintenance phase. In the setup phase, nodes organize themselves into clusters so that each cluster node will work as a cluster head itself. Deciding to become a cluster head within each node is done locally. On average, the percentage of the predetermined spatial location in each of the nodes serves as cluster head. The length of phase transitions is selected for each cluster head node of the cluster to collect data and, before sending it directly to the base station, the process of aggregating data is done. HEED [4] periodically selects $\mathrm{CHs}$ and builds equal clusters according to a hybrid of the residual energy of nodes and a secondary parameter. UCS [5] is the first proposed algorithm to resolve the problem of unbalanced energy dissipation by forming unequal clusters. EECS [6] and EDUC [7] are unequal cluster-based algorithms for WSNs in which the CHs transmit data to the BS by one-hop communication. EECS is proposed for homogeneous WSNs, it elects some tentative CHs randomly and utilizes a cluster head competition method to choose $\mathrm{CHs}$ from tentative $\mathrm{CHs}$, after that, each ordinary node selects $\mathrm{CH}$ basing on the distance from itself to $\mathrm{CH}$ and the distance from $\mathrm{CH}$ to the BS to construct unequal clusters. EDUC is proposed for heterogeneous WSNs. In [8] Mr.JanYue and colleagues have offered an EEBCDA algorithm. In this algorithm, the network environment as well as the swim lane is divided in sections of the size of the swim lane size, length and width are equal. Each swim lane, also called grid, is divided into smaller parts. Grid size is different in each swim lane. The greater the distance from the BS, the larger is the grid size. The grid node with the highest energy is selected as the cluster head node. This type of fencing can have a grid that contains a number of nodes with greater distance from the BS. As a result, a greater number of nodes become cluster-heads. This results in balancing the energy consumption of nodes and improving the network lifetime.

\section{EXPRESSION OF PROPOSAL}

The main issue of this paper is to introduce a routing method to enhance and improve the lifetime of sensor nodes and, thus, increase the lifetime of sensor networks. The main steps are divided into three stages. In the first stage, the network is divided into sections that can uniform energy consumption among sensor nodes. A heterogeneous distribution of nodes for this purpose is introduced. Secondly, a clustering algorithm for data aggregation is presented. And in the third phase, a multi-step hierarchical tree for sending aggregated data from nodes to the base station is presented. 


\subsection{A Heterogeneous Distribution of Nodes}

The initial energy of heterogeneous nodes is not equal. For this purpose, three types of nodes with different initial energies are considered. The distance between the BS is divided into three areas: The closest node to node with the lowest energy is placed in the middle part of the average energy and the farthest node with the highest energy content of each. Since nodes consume far more energy to send data to this node, energy consumption becomes more balanced and cause the nodes to die almost simultaneously.

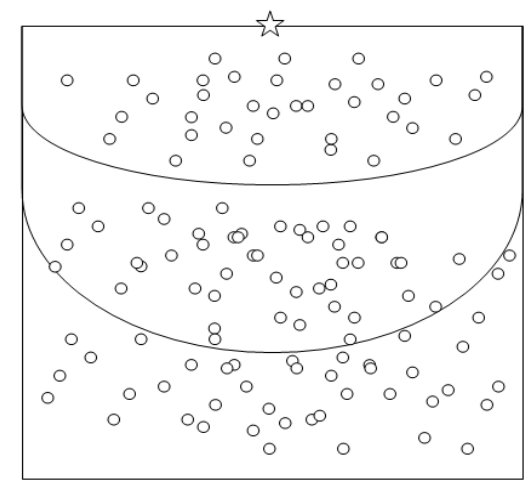

Figure 1.Network is divided into three zones

\subsection{Network Model}

To simplify the network model, we consider a WSN that $N$ heterogeneous nodes are uniformly dispersed within a square deployment area with border $B$. The left bottom vertex of deployment area locates at $\left(O \_x, O \_y\right)$ in Cartesian coordinate plane. In addition, we make a few assumptions: 1) the BS and all nodes are time synchronized and are stationary after deployment, the BS is located at $\left(B S \_x, B S \_y\right)$ which transmission power level according to the distance to the receiver; $3)$ the BS and all nodes are location-aware.

The first two assumptions are familiar in other algorithms. The third assumption is reasonable in many applications of WSNs in which the sensed data only make sense with location information, for other applications, the locations of BS and nodes can be easily obtained by utilizing localization algorithms. The time synchronization algorithms and localization algorithms are not discussed in our work.

\subsection{Energy Consumption Model}

We use the same energy consumption model used in EECS. The free space model is used if the distance between the transmitter and receiver less than a threshold $d 0$, otherwise, the multipath model is used. The energy spent for transmitting an $l$-bit message over distance $d$ is

$$
\begin{aligned}
& \operatorname{ETx}(l, d)=l \times \text { Eelec }+l \times \varepsilon f s \times d 2, d<d 0 \\
& l \times \text { Eelec }+l \times \varepsilon m p \times d 4, d \geq d 0
\end{aligned}
$$


where Eelec is the energy dissipated per bit to run the transmitter or the receiver circuit, $\varepsilon f s$ or $\varepsilon m p$, is the energy dissipated per bit to run the transmit amplifier. To receive this message, the expended energy is

$$
\operatorname{ERx}(l)=l \times \text { Eelec }
$$

The consumed energy of aggregating $m$ messages with $l$-bit is

$$
E A(m, l)=m \times l \times E D A
$$

where $E D A$ is the energy dissipated per bit to aggregate message signal.

\subsection{Problem Statement}

In the cluster-based WSNs in which the CHs transmit data to the BS by one-hop communication, there are three reasons leading to unbalanced energy dissipation: 1) a $\mathrm{CH}$ often spends more energy than a member;2) the amounts of received data of $\mathrm{CHs}$ are different; 3) the distances of transmitted data of $\mathrm{CHs}$ indifferent regions are different. Both LEACH and equal cluster-based algorithms are not able to balance the energy dissipation completely. Unequal cluster-based algorithms are considered as better solutions, they form clusters with unequal size according to the distance from each $\mathrm{CH}$ to $\mathrm{BS}$, expect that the $\mathrm{CH}$ further away from the $\mathrm{BS}$ has less members so that it is able to consume less energy to receive data and preserve more energy to transmit data. But the existing unequal cluster-based algorithms are deficient.

First of all, they do not consider the distribution of $\mathrm{CHs}$ in $\mathrm{CHs}$ rotation scheme, the selected $\mathrm{CHs}$ are randomly scattered in the network and the purpose of unequal clustering is affected. In addition, many unequal cluster-based algorithms make some ordinary nodes choose further $\mathrm{CHs}$ but not the closest $\mathrm{CHs}$ to form unequal clusters, so that these nodes have to spend excessive energy to transmit data.

\section{OUR PROPOSED METHOD}

Our proposed clustering method is also divided into rounds and every round consists of a set-up phase and a steady-state phase, especially, there is a network-division phase before the first round. The network is divided into rectangular regions firstly, called swim lanes, then, each swim lane is further partitioned into smaller rectangular regions, called grids. The node with maximal residual energy of each grid and the shortest distance to the BS is selected as $\mathrm{CH}$. The grids further away from the BS are bigger and have more nodes to participate in CHs rotation. In Fig 2, in which the dashed lines mark the division of swim lanes, the dotted-dashed lines denote the division of grids.

\subsection{Network-Division Phase}

Without loss of generality, we assume that the BS is above the deployment area along Y-axis. At first, the deployment area is divided into $S$ rectangular swim lanes along X-axis. All swim lanes 
have equal width $W$, and the length of each swim lane is equal to the border of deployment area. We use a sequence of integers from 1 to $S$ as the IDs of swim lanes, and the ID of the leftmost swim lane is 1 .

Then, each swim lane is partitioned into several rectangular grids along Y-axis. Each grid of each swim lane is assigned a level, we also use a sequence of integers starting from 1 as the levels of grids in each swim lane, and the level of the bottommost grid is 1. Each grid has the same width with swim lane. Both the number of grids and the length of each grid in a swim lane are related with the distance from the swim lane to BS. Our proposed method adjusts the size of each grid by setting its length. For different swim lanes, the further a swim lane is away from BS, the fewer grids it has. For same swim lane, the grid further away from the BS has longer length. We define an array $A$ with $S$ elements, in which the $k$-th element is the number of grids in swim lane $k$. Each grid is assigned a tuple $(i, j)$ as ID, which means that it is in swim lane $i$ and has level $j$. In addition, we define $S$ arrays to denote the lengths of grids, the $v$-th array $H v$ is the lengths of grids in swim lane $v$, and the $w$-th element $h v w$ of $H v$ is the length of grid $(v, w)$. The bounds of grid $(i, j)$ are

$O_{x}+(i-l) \times W<x \leq O_{x}+i \times W$

$O_{y}+\sum_{k=1}^{k \leq j-1} h_{i k}<y \leq O_{y}+\sum_{k=1}^{k \leq j} h_{i k}$

\subsection{Analysis}

Initially, the BS broadcasts a $B S \_M S G\left(\left(O \_x, O \_y\right),\left(B S \_x, B S \_y\right), B, W, S, A, H 1, \ldots, H S\right)$ message to all nodes and each node calculates the ID of the grid. At each stage of the implementation of the proposed method, a cluster head is selected for each grid. Between the nodes of a grid, as the cluster head node which has the highest residual energy is chosen. In the first round, all nodes is equal to the initial energy of all nodes close to the BS is selected as the cluster head. In subsequent rounds, if multiple nodes have the same energy, one that is closer to the BS as a cluster head is selected. In the first round by the cooperation of all nodes of a cluster head, the grid is selected. Initially, each node contains a message $N O D E \_M S G(k,(v, w), E r,(x$, $y)$ ) which is sent to other nodes on the grid. Where k: id nodes, (v, w): Number grid, Er: residual energy and node $(\mathrm{x}, \mathrm{y})$ : Location node. With this message, each node can get the information of rest of the nodes in a grid and then the node which has the highest residual energy is selected as the cluster head. After the first round, subsequent rounds of cluster head are selected by the cluster heads of the previous stage. In this case, the cluster head selects all nodes to send their data to the cluster head of the previous stage. The cluster heads steps before all nodes are sorted based on the residual energy and the new cluster head based on remaining energy level picks. To find information about all other nodes in the new cluster head, cluster head id message contains the previously selected cluster head sends information to all nodes in the grid. 


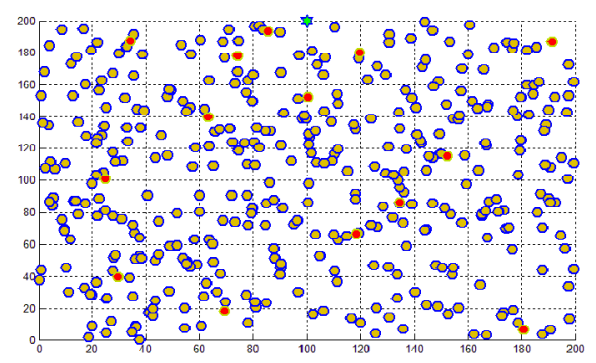

Figure 3. Displaying the nodes in the desired range and cluster heads are selected in the grid

\section{SIMULATION}

To simulate the algorithm, we examine two different environments :an area with a range of 200 to 200 meters in which 400 nodes are randomly placed, and an area with a range of 100 to 100 meters in which 100 nodes are randomly placed. Points on the bottom left to the top will be displayed as (O_x, O_y) and on the top right as (OW_x, OW_y). Consider the following assumptions for the network:

1) BS and the rest of the nodes in a fixed position, and BS nodes located outside the enclosure.

2) Distances between nodes are considered symmetric.

3) BS and all other nodes are aware of each other's location.

The initial model is shown in Figure 3, the network nodes are randomly placed in the desired range.

For network segmentation of 200 meters by 200 meters, the grid of the parameters is initialized as follows:

$\hat{S}=4 ; W=50 ; A=\{3,4,4,3\} ; H 1=H 4=\{100,70,30\} ; H 2=H 3=\{80,60,40,20\}$

Table 1. Parameters of simulation

\begin{tabular}{ll}
\hline parameter & value \\
\hline Number of nodes & 400 \\
Deployment area & $(0,0)-(200,200) \mathrm{m}$ \\
Location of BS & $(100,200) \mathrm{m}$ \\
Initial energy of & $0.5 \mathrm{~J}$ \\
each node & \\
$\mathrm{E}_{\text {elec }}$ & $50 \mathrm{~nJ} / \mathrm{bit}$ \\
$\varepsilon_{\mathrm{fs}}$ & $10 \mathrm{pJ} /\left(\mathrm{bit} \cdot \mathrm{m}^{2}\right)$ \\
$\varepsilon_{\mathrm{mp}}$ & $0.0013 \mathrm{pJ} /\left(\mathrm{bit} \cdot \mathrm{m}^{4}\right)$ \\
$\mathrm{d}_{0}$ & $87 \mathrm{~m}$ \\
$\mathrm{E}_{\mathrm{DA}}$ & $5 \mathrm{~nJ} /(\mathrm{bit} \cdot$ signal $)$ \\
Message size & $800 \mathrm{bit}$ \\
\hline
\end{tabular}


First, as shown in Figure 3, the nodes are placed randomly in the range considered. As can be seen from space BS, points (200 100) were chosen quite arbitrarily. The location of the BS is usually considered outside of the network. In the next phase, unequal division of the network into subnetworks, and the clustering is done. The simulation is done on various rounds and in each round based on the clustering of near nodes and the cluster head based on remaining energy level and takes close to the BS.Cluster heads are shown in Figure 3. The nodes in the cluster heads are red with green margins and all other nodes, the nodes are numerical. The number is the number of grid and cluster heads. As can be seen in Figure 4, 14 cluster heads are marked in different areas.

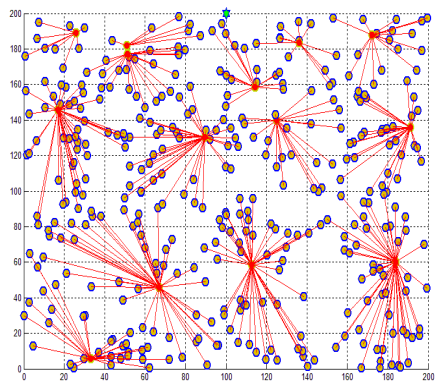

Figure 4.The proposed method of clustering

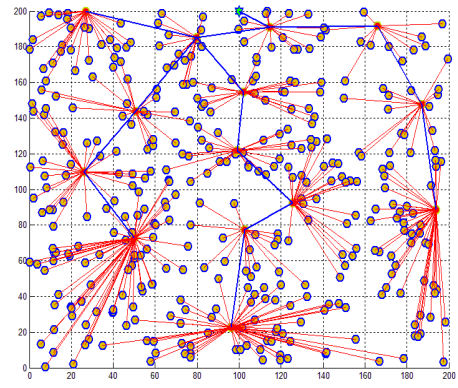

Figure 5. Hierarchical tree

After determining the cluster heads, the clustering is done. As can be seen, the clusters that are closer to the BS are smaller and the clusters that are further away are larger. These larger clusters have lower odds of being cluster head.

In this phase, Calculated for each non-cluster head node, the amount of energy to transmit data to the cluster head ETX-taking. And in each round, the value of their remaining energy is low. Figure 5 shows how to create a hierarchical tree where the blue lines indicate the relationship between the nodes.

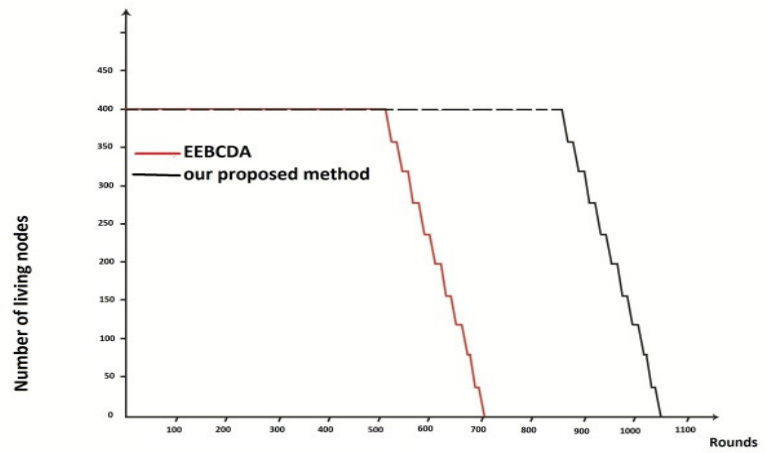

Fig 6. The number of living nodes over rounds 


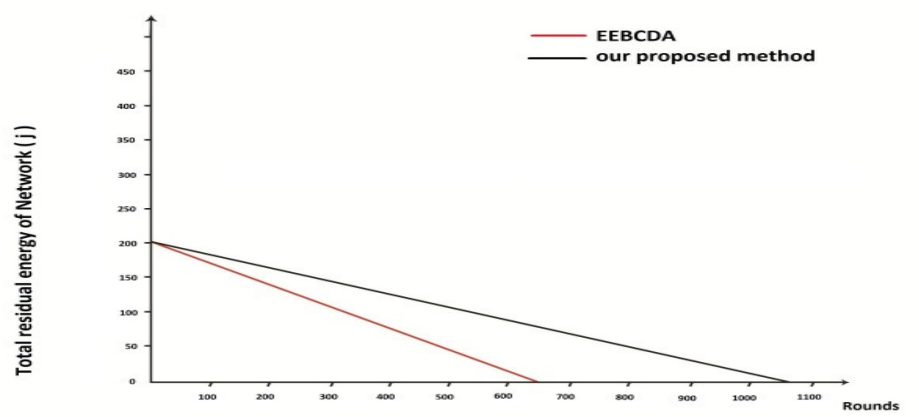

Fig 7.The total residual energy of network over rounds

First of all, we measure the lifetime of network. Fig 5 gives the number of living nodes over rounds. As evident from the figure, our proposed method has a longer network lifetime than EEBCDA. The first node of EEBCDA and our proposed method dies in the 591st round and 920th round, the last node dies in 698th round and 1058th round, respectively, our proposed method improves the network lifetime over by EEBCDA $23.56 \%$ and $13.12 \%$. Secondly, we compare the energy dissipation of our proposed method and EEBCDA. We take statistics of the total residual energy of network over rounds, as shown in Fig 5. It is explicit that our proposed method has more residual energy than EEBCDA in every same round, which intuitively illuminates that our proposed method is more energy efficient than EEBCDA. The ratio of time interval between the time when the first node dies and the time when the last node dies to the full time of network is able to indicate the balanced extent of energy dissipation, and the algorithm with smaller ratio has a better performance in aspect of balancing energy dissipation. The result of contrast shows that our proposed method is able to achieve more balanced energy dissipation than EEBCDA.

The next criterion for comparison is the number of packets being transferred to the BS in the simulation modeling process. Since the curve of the other available methods of data transfer depending on the total number of different methods are shown in table 2.As can be seen from these criteria, the proposed method is better.

Table 2.The number of packets transmitted

\begin{tabular}{|l|l|}
\hline Name of method & $\begin{array}{l}\text { The number of } \\
\text { packetstransmitted }\end{array}$ \\
\hline EEBCDA & 12000 \\
\hline Our proposed method & 18000 \\
\hline
\end{tabular}

\section{CONCLUSION}

In this paper, we focus on the problem of unbalanced energy dissipation in cluster-based and propose a novel cluster-based data aggregation protocol. The proposed method has been tried by a heterogeneous distribution of node energy consumption of nodes and network partitioning becomes more balanced and their lifetime is quite similar to that carried out this evaluation was realized. By creating a hierarchical structure, we optimize and minimize the energy consumption 
of nodes. Finally, when the new approach is used in the MATLAB simulation of the proposed method, we are able to obtain acceptable results.

\section{REFERENCES}

[1] Akyildiz IF, Su W, Sankarasubramaniam Y, Cayirci E. A survey on sensor networks. IEEE Commun Mag, 2002, 40(8), p.102-114.

[2] Rajagopalan R, Varshney PK. Data-aggregation techniques in sensor networks: a survey. IEEE Commun Surv \& Tutor, 2006,8(4), p. 48-63.

[3] Heinzelman WB, Chandrakasan AP, Balakrishnan H. Energy-efficient communication protocol for wireless microsensor networks. In Proc of IEEE Conf on System Sciences, 2000, p. 3005-3014.

[4] Younis O, Fahmy S. HEED: a hybrid, energy-efficient, distributed clustering approach for ad hoc sensor networks. IEEE Trans on Mobile Computing, 2004, 3(4), p. 366-379.

[5] Soro S, Heinzelman WB. Prolonging the lifetime of wireless sensor networks via unequal clustering. In Proc of IEEE Parallel and Distributed Processing Symposium, 2005, p. 1-8.

[6] Ye M, Li C, Chen G, Wu J. An energy efficient clustering scheme in wireless sensor networks. Ad Hoc \& Sensor Wireless Networks, 2006, 3, p. 99-119.

[7] Yu J, Qi Y, Wang G. An energy-driven unequal clustering protocol for heterogeneous wireless sensor networks, J Control Theory Appl, 2011, 9(1), p. 133-139.

[8] Yue J, Zhang W, Xiao W, Tang D, Tang J. Energy Efficient and Balanced Cluster-Based Data Aggregation Algorithm for Wireless Sensor Networks. International Workshop on Information and Electronics Engineering (IWIEE), 2012,2,p.2009-2015.

\section{AUTHORS}

\section{Shideh Sadat Shirazi}

Shideh Sadat Shirazi was born on 20 September 1986 in Tehran, Iran. She has been studying Computer Sciences at Islamic Azad University in Qazvin, Iran since 2011. One of the focuses of her studies are wireless sensor networks, with a special interest in energy efficiency. This paper represents an excerpt of her works in this field.

\section{Aboulfazl Torqi Haqiqat}

Abdoulfazl Torqi Haqiqat is Assistant Professor, Ph.D. in Computer Engineering at Islamic Azad University in Qazvin, Iran. He is an expert in the fields of high speed computer networks, wireless and mobile networks, i.e. MANET and sensor networks, distributed systems and distributed operating systems, computational intelligence. His interests also include neural networks, genetic algorithms, ant colonies, taboo searches, robotics, modeling, simulation and performance evaluation of computer systems and

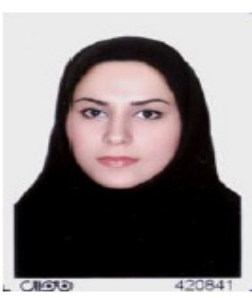
queuing theory. 